Proceedings

\title{
Review of LiDAR Sensor Data Acquisition and Compression for Automotive Applications ${ }^{\dagger}$
}

\author{
Ievgeniia Maksymova ${ }^{1}$, Christian Steger ${ }^{2}$ and Norbert Druml 1,* \\ 1 Infineon Technologies AG, 8020 Graz, Austria; ievgeniia.maksymova@infineon.com \\ 2 Institute of Technical Informatics, Graz University of Technology, 8010 Graz, Austria; steger@tugraz.at \\ * Correspondence: norbert.druml@infineon.com; Tel.: +43-517-775-796 \\ + Presented at the Eurosensors 2018 Conference, Graz, Austria, 9-12 September 2018.
}

Published: 6 December 2018

\begin{abstract}
Due to specific dynamics of the operating environment and required safety regulations, the amount of acquired data of an automotive LiDAR sensor that has to be processed is reaching several Gbit/s. Therefore, data compression is much-needed to enable future multi-sensor automated vehicles. Numerous techniques have been developed to compress LiDAR raw data; however, these techniques are primarily targeting a compression of 3D point cloud, while the way data is captured and transferred from a sensor to an electronic computing unit (ECU) was left out. The purpose of this paper is to discuss and evaluate how various low-level compression algorithms could be used in the automotive LiDAR sensor in order to optimize on-chip storage capacity and link bandwidth. We also discuss relevant parameters that affect amount of collected data per second and what are the associated issues. After analyzing compressing approaches and identifying their limitations, we conclude several promising directions for future research.
\end{abstract}

Keywords: LiDAR parameters; low-level data compression; compressed sensing

\section{Introduction}

Automotive LiDAR (Light Detection and Ranging) sensors will become a key element in self-driving cars, as they could provide valuable information under conditions that other surrounding observing sensors are not sensitive to. Its operational principle, Time-of-Flight (ToF), provides distance information by measuring the travel time of emitted light and is described by the following equation:

$$
R=\frac{\mathrm{c} \Delta T}{2}
$$

where $R$-range [m], $c$-light propagation velocity $[\mathrm{m} / \mathrm{s}], 4 T$-round trip time [s].

There are several different LiDAR systems available for environment perception [1], however only few of them are applicable for a broader automotive market. Scanning LiDAR is one of the most commonly used systems in the vehicle safety field and consists of one or more light emitters (TX) and one or more reflected light detectors (RX) to cover the required field-of-view (FoV). While the type of TX is not very important for the data acquisition, our case study is based on LiDAR with a microelectromechanical mirror system (MEMS). This type of transmitter is considered as the most promising way to reduce a LiDAR system's form factor and price, while providing high performance at the same time [2].

Building components of RX chain, on the other hand, have a direct impact on the amount of received data. The receiver chain consists of a photo diode array using avalanche photo diodes or single photon photo diodes followed by trans-impedance amplifiers. Additionally, analog-to-digital 
converters (ADC) can be incorporated to a LiDAR's receiving path to increase its integration level and eliminate a potential need of external components.

Depending on the sensor's configuration, LiDAR systems generate millions data points per second, which equals to several GBits/sec of raw data that needs to be transferred to an ECU for a real-time 3D point cloud computing. This vast amount of data is a major issue for current and future hardware/software/interconnect implementations in cars. The significance of raw data handling is further increasing with integrating several LiDAR sensors within one car's infrastructure. The rest of the paper is organized as follows: Section 2 describes the main LiDAR parameters and their contribution in the amount of received data. In Section 3, data rate reduction approaches applicable for raw data handling are reviewed. Finally, Section 4 concludes this work.

\section{LiDAR Key Parameters}

The amount of received data $\left(N_{R x}\right)$ depends on various LiDAR parameters such as required image and range resolution, FoV frame size, number of frames per second, laser pulse repetition frequency (PRF) and $R X$ chain configuration: number of APD elements, $A D C$ sampling frequency $\left(f_{A D C}\right)$ and resolution. Analytically it is described by the following equation:

$$
N_{R X}=\frac{t_{\text {sample }}}{t_{A C D}} \cdot N_{A D C} \cdot N_{\text {pixel }} \cdot N_{o s} \cdot N_{F R}
$$

given $t_{\text {sample }}-$ maximum pixel sampling time [s], $t_{A C D}=1 / f_{A D C}-A D C$ sampling period [s], $N_{A D C}-A D C$ resolution [bits/sample], $N_{\text {pixel }}-$ FoV frame size, Nos-system oversampling factor, $N_{F R}$-system frame rate.

The range resolution $(d \mathrm{R})$ or range measurement accuracy is the key parameter for choosing a sampling frequency for $A D C$, as it defines the minimum possible distance between adjacent targets that is resolvable by the sensor, and can be defined as $d \mathrm{R}=\mathrm{c}_{-} t_{A D C} / 2$.

Field of View $(\mathrm{FoV})$ is usually specified as a set of horizontal and vertical angles around the axis that is perpendicular to the sensor aperture, where the distance can be measured. The FoV frame size is a product of these angle values and the required image resolution.

Unlike an airborne LiDAR that gathers geospatial information, an automotive sensor has to deal with real-time dynamic surroundings information; therefore, its frame rate $\left(N_{F R}\right)$ has to be high enough to capture any moving object or approaching obstacle within its field of view. This aspect has to be considered while selecting PRF for a mobile LiDAR. An obvious choice for the frame rate is 25 or higher fps (frames per second) that is used in video recording.

The maximum PRF represents a number of emitted pulses per second and determines the point density at a targeted frame rate, and it is an important parameter of LiDAR systems as the costs for the lasers are mainly driven by the PRF.

The system oversampling factor defines how many times a pixel should be sampled within a single frame. Multiple samples of the pixel could be processed with various algorithms such as histogramming or averaging. Moreover, by applying a threshold to the processed oversampled data noise (e.g., sunlight interference) can be removed from the pixel data and, thus, the sensor SNR will be improved.

\section{Data Rate Reduction}

Nowadays there are various techniques available for LiDAR data compression. Among others are aforementioned histogramming, LASzip and the real-time point cloud compression [3]. Many of these algorithms require an entire frame to be completed and transferred from a sensor to ECU before compression can start. This implies that the compression is only beneficial at the point-cloud computing level, which is advantageous for airborne LiDAR but not as much for the mobile one due to different dynamics of received information. Thus, data compression should be applied already during data receiving for optimizing sensor's storage and link capacity. In order to make it 
possible one should turn attention to low-level compression approaches, potential application scenarios or advanced data capturing techniques (e.g., compressed sensing).

\subsection{Low-Level Data Compression}

There is one requirement that has to be kept in mind when selecting a data compression algorithm for on-chip data storage optimization: the encoding efficiency has to be constant over any $\mathrm{N}$ samples. In case it cannot be completely fulfilled, one might also explore advanced or customized memory access patterns. The link load reduction, on the other hand, requires a fast encoding process and a high compression ratio. In this sub-section we mention several low-level lossless data compression approaches that could be considered for data compression to optimize on-chip data storage and/or reduce the link load between the sensor and ECU.

Binary arithmetic coding is a subset of the statistical lossless arithmetic coding algorithm [4] that deals only with ' 0 ' and ' 1 ', which allows it to be implemented in hardware. Despite the compression algorithm's simplicity, it is rather slow and would require clock frequencies of several $\mathrm{GHz}$ to compress LiDAR's data stream; however, it might be of use for optimizing the on-chip data storage as statistical manipulations over a limited amount of bits could be done rather fast.

LZMA or Lempel-Ziv-Markov chain algorithm [5] is a lossless dictionary-based compression method originated from LZ77 [6] that uses a combination of a sliding window algorithm and dynamic dictionary to compress unknown data and enhanced with delta filter and a range coder. In [5] authors implemented LZMA on FPGA and achieved over 50\% compression ratio. As these algorithms are dictionary-based, there might be some limitations with respect to on-chip storage optimization: an encoding efficiency that has to be constant over any M samples, and the dictionary has to be stored next to a compressed data; Nevertheless, it could be explored for compressing of a raw data stream.

The Golomb-Rice encoding (GR) is another lossless data compression technique [7] that is widely used in several lossless image and audio codecs, because the decoding process doesn't require a transfer of explicit coding tables. The encoding process is based on the probability of the values, where small numbers are assumed more likely to be present in the data stream than big ones. The relation between size and probability is captured in a parameter, called the divisor. Under ideal weather conditions most of LiDAR raw data could be considered as an ambient noise, which has smaller magnitude comparing to target returns. Thus, GR coding is a good candidate for compressing the raw data stream. Moreover, as it was presented by Moussalli et al. in [8] hardware FPGA based implementation of the algorithm is capable of encoding several Gsps and, therefore, should be further evaluated as a part of LidAR raw data handling algorithm.

\subsection{Compressed Sensing}

In recent years a new concept of sampling was proposed by Candence and Wakin [9]. This new approach challenged the Nyquist theorem and showed that it's not a necessary condition for signal reconstruction. Compressed sensing (CS) is based on the idea that if a signal is sparse in a sampling domain and incoherent with respect to information representation domain then it is possible to sample that signal at a slower rate than the Nyquist limit and reconstruct it with a certain precision. It has been shown in $[10,11]$ that CS could be effectively applied to scanning LiDAR systems and might change the way data is acquired by reducing number of required measurements within a frame and provides very high compression ratios. However, up to the best knowledge of the authors, these results are analytical and based on various MATLAB simulations.

MEMS-based LiDAR scans the environment non-uniformly, which could be considered as both an advantage and a challenge for applying CS algorithms. The advantage refers to a randomized sampling process of the frame, while the main challenge occurs in adapting $R X$ chain to select and acquire samples that are sufficient for keeping the received data sparse. Moreover, automotive LiDAR sensors should process the data in real-time, which could be a blocking point for some CS algorithms with respect to decompression and reconstruction speed. Thus, further research in this field is required before CS could be successfully applied to the automotive sensor. 


\section{Conclusions}

The analysis of low-level compression algorithms showed that some of them could be applicable for optimizing on-chip memory capacity for data acquisition, despite limitations of achieving a deterministic compression ratio over any $\mathrm{N}$ samples. The combination of a low-level compression technique and a customized memory access pattern has been identified as one of directions for future research. Particularly of interest is GR algorithm due to its encoding speed and high compression ratio. The compressed sensing provides a possibility of data reconstruction without fulfilling the Nyquist theorem and, as it was shown in academia, could be effectively applied to LiDAR. The reported results are based on LiDAR that scans environment uniformly; however, our research targets a MEMS-based sensor, which implies a non-uniformed scanning of the surrounding. Therefore, another path for future research lies in exploring a possibility of implementing MEMSbased LiDAR with a modified data acquisition by incorporating compressed sensing.

Author Contributions: All authors contributed equally to the contents of this paper. I.M. wrote the paper.

Funding: This research was funded by the Austrian Federal Ministry for Transport, Innovation and Technology grant number 860032 .

Conflicts of Interest: The authors declare no conflict of interest. The founding sponsors had no role in the design of the study; in the collection, analyses, or interpretation of data; in the writing of the manuscript, and in the decision to publish the results.

\section{References}

1. Behroozpour, B.; Sandborn, P.; Ming, C. Wu; Boser, B. Lidar system architectures and circuits. IEEE Commun. Mag. 2017, 55, 135-142.

2. Innoluce. Laser Scanning Platform. Available online: http://www.innoluce.com/ (accessed on 18 June 2018).

3. Golla, T.; Klein, R. Real-time point cloud compression. In Proceedings of the 2015 IEEE/RSJ International Conference on Intelligent Robots and Systems (IROS), Hamburg, Germany, 28 September-2 October 2015.

4. Kuang, S.-R.; Jou, J.-M.; Chen, Y.-L. Design of an adaptive on-line binary arithmetic-coding chip. IEEE Trans. Circuits Syst. I Fundam. Theory Appl. 1998, 45, 693-706.

5. Leavline, E.J.; Singh, D.A. Hardware implemetation of lzma data compression algorithm. Int. J. Appl. Inf. Syst. 2013, 5, 51-56.

6. Ziv, J.; Lampel, A. A universal algorithm for sequential data compression. IEEE Trans. Inf. Theory 1977, 23, 337-343.

7. Rice, R. F.; Plaunt, J. Adaptive Variable-Length Coding for Efficient Compression of Spacecraft Television Data. IEEE Trans. Commun. Technol. 1971, 19, 889-897.

8. Moussalli, R.; Najjar, W.; Xi, L.; Khan, A. A high throughput no-stall golomb-rice hardware decoder. In Proceedings of the IEEE 21st Annual International Symposium on Field-Programmable Custom Computing Machines, Seattle, WA, USA, 28-30 April 2013.

9. Candes, J.E.; Walkin, B.M. An introduction to compressive sampling. IEEE Signal Process. Mag. 2008, $25,21-30$.

10. Martinez, J.E.C. Remote-Sensed Lidar Using Random Sampling and Sparse Reconstruction. Available online: http://wordpress.nmsu.edu/ccreuser/files/2014/07/castorena-ITC2011.pdf (accessed on 18 June 2018).

11. Joon, I.J.; Leonessa, A.; Kurdila, A.; Ryoo, Y.-J. A real-time data compression for ground-based 3d lidar data using wavelets and compressive sensing. In Proceedings of the Joint 5th International Conference on Soft Computing and Intelligent Systems and 11th International Symposium on Advanced Intelligent Systems, Okayama, Japan, 31 July 2010. 